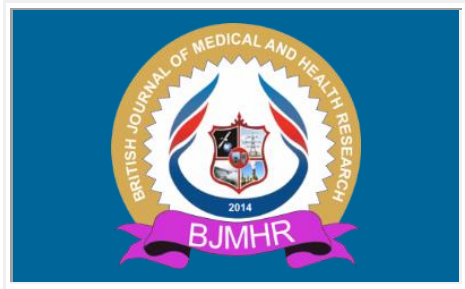

\title{
BJMHR
}

British Journal of Medical and Health Research

Journal home page: www.bjmhr.com

\section{Sleep Disorder Breathing in Obese Preschool Children: Case Series and Review of Literatures}

\author{
Abdullah Khayat \\ Department of Pediatric, College of Medicine, Taif University
}

\begin{abstract}
Obstructive sleep apnea (OSA) is a common problem estimated at 2-5\% in the school-aged children. As obesity prevalence is increasing universally, OSA is estimated to be higher than their lean children. Obesity related OSA is increasing in all age groups including younger children. The aim of this study is to focus on the importance of OSA among obese preschool children and to determine what risk factors are associated with OSA. A retrospective chart review for children $\leq 6$ years is identified from tertiary centre in Saudi Arabia who had a polysomnography (PSG). Obesity was defined by a BMI of > 95th percentile for age. OSA was diagnosed if the obstructive apnea hypopnea index (OAHI) was greater than 1 event per hour. There were 4 children identified; the mean age was 4.5 years meaning BMI was $28(\mathrm{SD} \pm 1.2)$. the prevalence of OSA in those children was high. Young obese children should be assessed for OSA. A history of snoring and daytime sleepiness should facilitate referral for a PSG, especially in resource-limited settings.
\end{abstract}

Keywords: Obesity. Obstructive sleep apnea. Sleep-disordered breathing. Pediatrics. 


\section{INTRODUCTION}

OSA is characterized by recurrent partial and/or complete obstruction of the upper airway and associated with episodes of intermittent nocturnal oxygen desaturations, and/or sleep disruption ${ }^{1}$. The prevalence of OSA in pediatric population is estimated $1-5 \%$ with adenoidal and tonsillar hypertrophy as the most common cause for such condition ${ }^{2}$. However, other clinical conditions like cranio-fascial abnormalities, neuromuscular problem and obesity increase the prevalence of $\mathrm{OSA}^{3}$. If left untreated, OSA in young children increase the prevalence of neurobehavioral problems, cardiovascular effect as well as metabolic disease and poor quality of life $\mathrm{e}^{4-6}$. The gold standard for the diagnosis of OSA is polysomnography (PSG) ${ }^{7}$. Other modalities for diagnosis of OSA in pediatric population is still under investigation ${ }^{8}$.

Obesity in young children is growing problem worldwide especially in developed countries with prevalence estimated to be $7 \%^{9}$. Many studies indicate that obesity-related comorbidities such as hypertension, diabetes, and obstructive sleep apnea are considered a significant concern $^{10,11}$. Obese individuals are at higher risk for development of OSA because of upper airway obstruction secondary to adenotonsillar hypertrophy. Other factors of upper airway obstruction are due to increased adiposity around the neck, pharynx, and the base of the tongue $^{12,13}$. Obesity-related OSA prevalence is estimated to occur in $25 \%$ of obese youth ${ }^{14}$. However, there are extremely limited data on the prevalence of obesity related OSA in very young children ${ }^{15}$.

The childhood obesity has significantly changed the scope of pediatric OSA and more data are needed to make this a priority to treat OSA in obese children.

\section{MATERIALS AND METHOD}

This study was a retrospective chart review for patient attended the sleep laboratory for an overnight PSG for evaluation of suspected OSA. Only obese patients less than 7 years were identified and included in the study. The body mass index (BMI) was calculated as weight (kg) divided by the height squared $\left(\mathrm{m}^{2}\right)$ which was measured at the time of $\mathrm{PSG}^{16}$. Obesity was defined by a BMI of $>95$ th percentile for age and gender or a z-score of $>2^{17}$. Four patients were identified using clinical history, physical examination, and sleep questionnaire.

PSG was performed and scored manually based on the American Academy of Sleep Medicine (AASM) guidelines ${ }^{18}$. Patients underwent a standard overnight PSG using Alice 6 (Philips, USA) Diagnostic sleep system with electro-oculogram, electroencephalogram - EEG, electromyogram, EMG, nasal air flow, chest belt for thoracic and abdominal movements, pulse oximetry, snoring, leg movements and analysis data acquisition and analysis systems. video and audio recordings were obtained as well as body position. The presence or absence of snoring was documented by direct observation by overnight sleep technologist. EEG registered 
with six electrodes. Sleep stages and architecture including total sleep time, sleep and REM onset latency, and time in each sleep stage were assessed by manual techniques. Respiratory events were recorded as obstructive apnea with nasal airflow cessation for minimum $10 \mathrm{sec}$ and hypopnea - reduction or air flow amplitude for $30 \%$ or more with desaturation at least $3 \%$ or microarousal at EEG.

The severity of OSA was determined by the obstructive apnea-hypopnea index (OAHI), the number of obstructive, mixed apneas, and obstructive hypopneas per hour during sleep. OSA was diagnosed if the OAHI was greater than 1 event per hour with severity range defined as mild, moderate, and severe if $<5$ events $/ \mathrm{h}, \geq 5-10$ events/h, and $>10$ events/h, respectively according to pediatric rules ${ }^{19}$. Patients' demographics information and polysomnographic data were presented as mean (standard deviation, SD) for continuous variables and number (percentage) for categorical variables. The study was approved by the Taif university Research Ethics Board.

\section{RESULTS AND DISCUSSION}

After reviewing the charts four children with obesity and symptoms suggestive of OSA were identified. All demographic data are summarized in Table-1. PSG findings at baseline are listed in Table-2.

\section{Case 1:}

A 4-year-old boy known case of moderate persistent asthma on inhaled corticosteroid, Chronic lung disease and obesity. He underwent to diagnostic sleep study to rule out OSA. the parents reported history of loud snoring and night sweating. His BMI was 28. In his PSG he had consolidated sleep and all the sleep stages were observed. He slept in all position. He had many respiratory events which included central and hypopnea events. He had also desaturation to $86 \%$ during NREM associated with respiratory event. His baseline $\mathrm{SaO} 2$ was $98 \%$ with OAHI

of 11.8 events per hour.

\section{Case 2:}

A 5-year-old boy with known case of obesity, history of night cough, snoring, gasping, nasal blockage, and mouth breathing. He underwent overnight diagnostic sleep study. His BMI was 27. His PSG showed fragmented sleep with poor sleep efficiency. He had paradoxical breathing and flow limitation. He had frequent respiratory events during REM stage. He had more in supine position. He had evidence of hypoventilation. His baseline oxygen saturation was $95 \%$ with a minimum value of $65 \%$. Baseline $\mathrm{CO}_{2}$ was $44 \mathrm{mmHg}$ with a maximum of $46 \mathrm{mmHg}$. The total apnea-hypopnea index was 16.7 events/hr.

\section{Case 3:}


A 4-year-old boy known as obesity underwent diagnostic sleep study to rule out OSA. His parents noted snoring and mouth breathing. He has symptoms mimic attention deficit hyperactivity disorder ADHD. His BMI was 32. He had fragmented sleep and all sleep stages were observed. EMG chin activation was also observed. He has sweating and he had respiratory events with $3 \%$ desaturation. The total apnea-hypopnea index was 11.4 events/hr.

\section{Case 4:}

A 6-year-old boy known case of Obesity possible asthma and allergic rhinitis. His BMI was 29. He underwent overnight PSG. He had consolidated sleep and all sleep stages were observed. EMG chin activation was also observed. He had snoring. $\mathrm{His} \mathrm{CO}_{2}$ was ranged from 42-45 $\mathrm{mmHg}$ and his minimum oxygen saturation was $91 \%$. He slept in all positions. The total apnea-hypopnea index was 2.6 events/hr.

\section{DISCUSSION:}

The prevalence of obesity is growing all over the world. OSA also not recognized cause of morbidity and mortality especially in developing countries. This case series showed that OSA is highly prevalent in those obese young children. Data regarding this situation are lacking as there are only very few studies that showed the prevalence of OSA in preschool children. This most likely because of lacking expertise of sleep physician and technicians, challenges in behaviour of children so younger children will need to have a PSG done in a specialized pediatric sleep lab. This considered a major problem worldwide to get a proper facility. In Canada, for example, the waiting times is up to 2 years to get a proper PSG. In Saudi Arabia, there are few sleep centers taking care of pediatric population.

Most of those children were referred to sleep lab because of nighttime symptoms, mostly snoring and mouth breathing. However, other symptoms may be present during the interview. Snoring In this series one child had symptoms suggestive of ADHD. Interestingly, based on other studies they showed that those children with OSA were more sleepy than obese subjects who did not have OSA.

Bin-hasan et al estimated the prevalence of OSA in young children to be around 36\%, however it was a retrospective study, relatively small cohort, and highly selected patient. Mallory et al. reported $24 \%$ of obese children had OSA. In older children two cohort with control group showed high prevalence of OSA in children with obesity. Kheirandish-Gozal et al found OSA in $78 \%$ of overweight/obese with history of habitual snoring and OAHI around 10 events/hr which is in severe range ${ }^{20}$. Raede et al reported OSA in 54\% obese vs $30 \%$ non-obese ${ }^{14}$. Many other Cohort without control showed significant prevalence of OSA in obese children and ranged between $24-64 \%{ }^{10,21-24}$. 
Many factors play significant rules in development of OSA in children in general. The most common cause of OSA in children is adeno-tonsillar hypertrophy. This fact is the same for those children with obesity ${ }^{3,25}$. In study by Arens et al it showed obese children with OSA compared with non OSA had a significantly larger volume of adenoids, palatine tonsils, and retropharyngeal lymph nodes. Also, obese subjects with OSA, when compared children without OSA, had significantly larger parapharyngeal fat pads and increased abdominal visceral fat suggesting that these are additional risk factors for obesity related OSA ${ }^{26}$.

If left untreated, those children will develop future complications which include cardiovascular dysfunction like hypertension and endothelial dysfunction. Systemic Inflammation, metabolic derangements, neurocognitive behavioural disorders, and poor quality of Life.

\section{CONCLUSION:}

In summary, sleep disordered breathing is very common in young obese children. Early identification of this problem is critical to refer them to pediatric specialist for detailed history, physical examination, and overnight polysomnography. If left untreated many complications will developed including cardiac and neurological deficits.

\section{REFERENCES:}

1. Bradley TD, Floras JS. Obstructive sleep apnoea and its cardiovascular consequences. The Lancet. 2009;373(9657):82-93.

2. Lumeng JC, Chervin RD. Epidemiology of pediatric obstructive sleep apnea. Proceedings of the American Thoracic Society. 2008;5(2):242-252.

3. Dayyat E, Kheirandish-Gozal L, Capdevila OS, Maarafeya MMA, Gozal D. Obstructive sleep apnea in children: relative contributions of body mass index and adenotonsillar hypertrophy. Chest. 2009;136(1):137-144.

4. Tauman R, O'Brien LM, Ivanenko A, Gozal D. Obesity rather than severity of sleepdisordered breathing as the major determinant of insulin resistance and altered lipidemia in snoring children. Pediatrics. 2005;116(1):e66-e73.

5. Beebe DW, Ris MD, Kramer ME, Long E, Amin R. The association between sleep disordered breathing, academic grades, and cognitive and behavioral functioning among overweight subjects during middle to late childhood. Sleep. 2010;33(11):14471456.

6. Amin R, Somers VK, McConnell K, et al. Activity-adjusted 24-hour ambulatory blood pressure and cardiac remodeling in children with sleep disordered breathing. Hypertension. 2008;51(1):84-91.

7. Aurora RN, Zak RS, Karippot A, et al. Practice parameters for the respiratory indications for polysomnography in children. Sleep. 2011;34(3):379-388. 
8. Marcus CL, Brooks LJ, Ward SD, et al. Diagnosis and management of childhood obstructive sleep apnea syndrome. Pediatrics. 2012;130(3):e714-e755.

9. Ogden CL, Carroll MD, Kit BK, Flegal KM. Prevalence of obesity and trends in body mass index among US children and adolescents, 1999-2010. Jama. 2012;307(5):483490.

10. Li AM, Au CT, Sung RYT, et al. Ambulatory blood pressure in children with obstructive sleep apnoea: a community based study. Thorax. 2008;63(9):803-809.

11. Buchwald H, Estok R, Fahrbach K, et al. Weight and type 2 diabetes after bariatric surgery: systematic review and meta-analysis. The American journal of medicine. 2009;122(3):248-256.

12. Schwab RJ, Gupta KB, Gefter WB, Metzger LJ, Hoffman EA, Pack AI. Upper airway and soft tissue anatomy in normal subjects and patients with sleep-disordered breathing. Significance of the lateral pharyngeal walls. American journal of respiratory and critical care medicine. 1995;152(5):1673-1689.

13. Schwab RJ, Pasirstein M, Pierson R, et al. Identification of upper airway anatomic risk factors for obstructive sleep apnea with volumetric magnetic resonance imaging. American journal of respiratory and critical care medicine. 2003;168(5):522-530.

14. Reade EP, Whaley C, Lin J-J, McKenney DW, Lee D, Perkin R. Hypopnea in pediatric patients with obesity hypertension. Pediatric Nephrology. 2004;19(9):1014-1020.

15. Bin-Hasan S, Katz S, Nugent Z, et al. Prevalence of obstructive sleep apnea among obese toddlers and preschool children. Sleep and Breathing. 2018;22(2):511-515.

16. Cole TJ, Bellizzi MC, Flegal KM, Dietz WH. Establishing a standard definition for child overweight and obesity worldwide: international survey. Bmj. 2000;320(7244):1240.

17. Hedley AA, Ogden CL, Johnson CL, Carroll MD, Curtin LR, Flegal KM. Prevalence of overweight and obesity among US children, adolescents, and adults, 1999-2002. Jama. 2004;291(23):2847-2850.

18. Berry RB, Gamaldo CE, Harding SM, et al. AASM scoring manual version 2.2 updates: new chapters for scoring infant sleep staging and home sleep apnea testing. American Academy of Sleep Medicine; 2015.

19. Iber C, Ancoli-Israel S, Chesson AL, Quan SF. The AASM manual for the scoring of sleep and associated events: rules, terminology and technical specifications. vol 1. American academy of sleep medicine Westchester, IL; 2007.

20. Kheirandish-Gozal L, Capdevila OS, Kheirandish E, Gozal D. Elevated serum aminotransferase levels in children at risk for obstructive sleep apnea. Chest. 2008;133(1):92-99. 
21. Verhulst SL, Schrauwen N, Haentjens D, et al. Sleep-disordered breathing in overweight and obese children and adolescents: prevalence, characteristics and the role of fat distribution. Archives of disease in childhood. 2007;92(3):205-208.

22. Mallory Jr GB, Fiser DH, Jackson R. Sleep-associated breathing disorders in morbidly obese children and adolescents. The Journal of pediatrics. 1989;115(6):892-897.

23. Barone JG, Hanson C, DaJusta DG, Gioia K, England SJ, Schneider D. Nocturnal enuresis and overweight are associated with obstructive sleep apnea. Pediatrics. 2009;124(1):e53-e59.

24. Shen Y, Xu Z, Shen K. Urinary leukotriene E4, obesity, and adenotonsillar hypertrophy in Chinese children with sleep disordered breathing. Sleep. 2011;34(8):1135-1141.

25. Redline S, Storfer-Isser A, Rosen CL, et al. Association between metabolic syndrome and sleep-disordered breathing in adolescents. American journal of respiratory and critical care medicine. 2007;176(4):401-408.

26. Arens R, Sin S, Nandalike K, et al. Upper airway structure and body fat composition in obese children with obstructive sleep apnea syndrome. American journal of respiratory and critical care medicine. 2011;183(6):782-787.

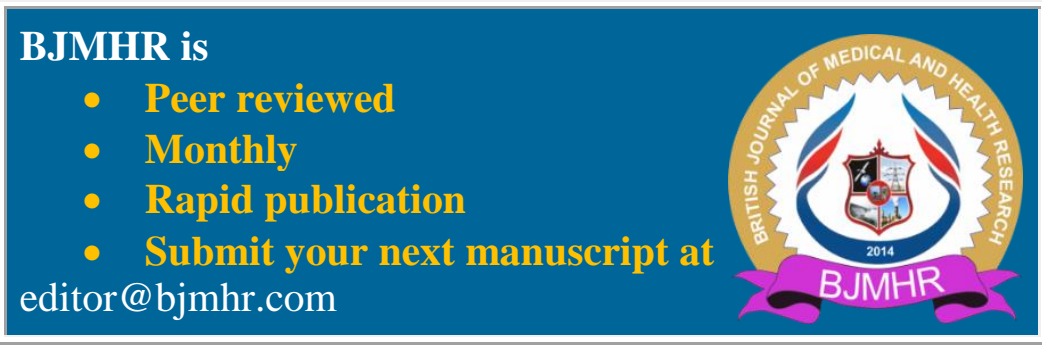

